\title{
Snail overexpression induces an epithelial to mesenchymal transition and cancer stem cell-like properties in SCC9 cells
}

\author{
Li-Fang Zhu ${ }^{1,2}$, Yong $\mathrm{Hu}^{3}$, Cong-Chong Yang ${ }^{1,2}$, Xiao-Hui Xu ${ }^{1,4}$, Tian-Yun Ning ${ }^{1,2}$, Zi-Lu Wang ${ }^{1}$, Jin-Hai Ye ${ }^{1,5}$ \\ and Lai-Kui Liu' ${ }^{1,2}$
}

Local invasiveness and distant metastasis are critical factors that contribute to oral squamous cell carcinoma-related deaths. Increasing evidence has shown that the epithelial to mesenchymal transition (EMT) is involved in cancer progression and is associated with the 'stemness' of cancer cells. Snail is a transcriptional factor that can induce EMT and preserve stem-cell function, which may induce resistance to radio- and chemotherapies in the cells. In the present study, SCC9 cells were transfected with an empty vector or a vector encoding human Snail (SCC9-S). Overexpression of Snail induced SCC9 cells to undergo EMT, in which the cells presented a fibroblast-like appearance, downregulated the epithelial markers E-cadherin and $\beta$-catenin, upregulated the mesenchymal marker vimentin, and associated with highly invasive and metastatic properties. Furthermore, the induction of EMT promoted cancer stem cell (CSC)-like characteristics in the SCC9-S cells, such as low proliferation, self-renewal, and CSC-like markers expression. These results indicate that overexpression of Snail induces EMT and promotes CSC-like traits in the SCC9 cells. Further understanding the role of Snail in cancer progression may reveal new targets for the prevention or therapy of oral cancers.

Laboratory Investigation (2012) 92, 744-752; doi:10.1038/labinvest.2012.8; published online 20 February 2012

KEYWORDS: cancer stem cell; EMT; oral squamous cell carcinoma; overexpression; Snail

Oral squamous cell carcinoma (OSCC) is the most frequent type of cancer in the oral cavity and is associated with high morbidity and poor prognosis. ${ }^{1,2}$ Despite progress in surgery, chemotherapy, and radiotherapy, the 5-year survival rate has remained at $50-55 \%$ over the past several decades. ${ }^{3}$ Local or regional recurrences and distant metastases have a critical role in this process, and the mechanism underlying their occurrence remains poorly understood. ${ }^{2,4}$

During metastatic progression, tumour cells lose cell-cell adhesion, detach from the primary site, invade the basement membrane, survive and circulate in the blood vessels, leave the bloodstream, and finally colonise in a new host environment to form micrometastases. ${ }^{2,5-7}$ A growing body of research strongly suggests that the epithelial to mesenchymal transition (EMT), which occurs normally during embryonic development, tissue remodelling, and wound healing, is a critical early event in tumour invasion and metastasis. ${ }^{8-10} \mathrm{It}$ is characterised by downregulation of epithelial markers, such as E-cadherin, and upregulation of mesenchymal markers, such as vimentin. During the process of EMT, epithelial cells acquire mesenchymal cell properties and show reduced intercellular adhesion and increased invasion. ${ }^{11}$

The transcriptional repressor Snail, which is a zinc finger protein, first described in Drosophila melanogaster, can bind to the E-boxes in the human E-cadherin promoter and suppress its transcription. ${ }^{12,13}$ Snail has previously been implicated in triggering EMT during embryonic development, fibrosis, and tumour progression. ${ }^{14}$ This process also occurs in the progression of carcinomas (including oral carcinoma cells), following the downregulation of E-cadherin expression or co-expression of NBS1. ${ }^{13,15-17}$ Snail also has been linked to cell survival or apoptosis, cell polarity, cancer cell invasion,

${ }^{1}$ Department of Basic Science of Stomatology, Institute of Stomatology, Nanjing Medical University, Nanjing, People's Republic of China; ${ }^{2}$ Department of Basic Science of Stomatology, College of Stomatology, Nanjing Medical University, Nanjing, People's Republic of China; ${ }^{3}$ Department of Stomatology, Suzhou Kowloon Hospital Shanghai Jiaotong University Medical School, Suzhou, People's Republic of China; ${ }^{4}$ Department of the First Outpatient, College of Stomatology, Nanjing Medical University, Nanjing, People's Republic of China and ${ }^{5}$ Department of Oral and Maxillofacial Surgery, College of Stomatology, Nanjing Medical University, Nanjing, People's Republic of China

Correspondence: Dr L-K Liu, PhD, Department of Basic Science of Stomatology, College of Stomatology, Nanjing Medical University, Postal\#210029 $136 \#$ Hanzhong Road, Nanjing, Jiangsu, People's Republic of China.

E-mail: laikuiliu@126.com

Received 23 June 2011; revised 19 December 2011; accepted 21 December 2011 
recurrence, and the acquisition of cancer stem cell (CSC)-like characteristics. $^{14,18,19}$

Increasing evidence suggests that tumours develop and progress from a small subset of cells with the ability to selfrenew, which enter the cell cycle infrequently. They constitute a population that is intrinsically resistant to classic treatments that are designed to kill cycling cells. ${ }^{20}$ Such cells are called CSCs and have been identified in many cancers, including colon, breast, liver, brain, prostate, pancreatic, head and neck, lung, and hematopoietic malignancies. ${ }^{14,21}$

A relationship between the EMT and CSCs has been observed, with the evidence suggesting that EMT cells acquire stem cell-like traits and that CSCs exhibit a mesenchymal-like appearance in immortalised nontumourigenic mammary epithelial cells and breast cancers. ${ }^{22}$ Furthermore, CSCs have been identified in head and neck SCC. It has been shown that head and neck SCC contains a subpopulation of cancer cells that have self-renewing ability, form new tumours, and possess the properties of CSCs. ${ }^{4}$ However, an association between the EMT and CSCs induced by Snail in the SCC9 cells has not been examined previously.

Taking this evidence together, we insight into the mechanisms that overexpression of Snail induces cells to undergo the EMT and gain stem cell-like characteristics in OSCC. Investigating the role of Snail at the molecular level in this process may identify novel treatment strategies against the most common malignancy of the oral cavity.

\section{MATERIALS AND METHODS}

\section{Cell Culture, Plasmid Construction, and Transfection}

The human SCC9, which is derived from carcinoma of the tongue, was obtained from the American Type Culture Collection (ATCC, Manassas, VA, USA). Cells from this line were maintained in a 1:1 mixture of Dulbecco's modified Eagle's medium and Ham's F12 medium (Invitrogen, Burlington, Ontario, Canada) supplemented with $10 \%$ fetal bovine serum (FBS, Invitrogen), $400 \mathrm{ng} / \mathrm{ml}$ hydrocortisone (Sigma-Aldrich, St Louis, MO, USA) and antibodies (Invitrogen). A fulllength cDNA for human Snail based on the published sequence (NM_005985.2) was amplified by RT-PCR, with the incorporation of Hind III (TaKaRa, Otsu, Shiga, Japan) and Bam HI (TaKaRa) restriction sites in the primers. After agarose gel electrophoresis, the fragment was recovered by gel extraction (OMEGA, Norcross, GA, USA) and ligated to the pMD18-T vector (TaKaRa). The expected PCR product was confirmed by sequencing. The entire coding sequence was then excised from the pMD18-T vector by digestion with Hind III and Bam $\mathrm{HI}$ and subcloned into the mammalian expression vector pEGFP-N1 (kindly provided by $\mathrm{Zi}-\mathrm{Lu}$ Wang, Number U55762). The final construct was verified by sequencing. Positive clones were selected by kanamycin and amplified in bacterial culture media. All the plasmids were purified using the Endo-free Plasmid Mini Kit II (OMEGA). For transfection, the SCC9 cells were plated in six-well plates (Corning, Lowell, MA, USA), cultured in a complete growth medium to $80 \%$ confluency, and then cultured in the medium without FBS for $12-16 \mathrm{~h}$. The transfection was performed using $2 \mu \mathrm{g}$ (per dish) of either pEGFP-N1 or pEGFP-N1-Snail and Lipofectamine 2000 (Invitrogen) reagent, according to the manufacturer's protocol. At $48 \mathrm{~h}$ post-transfection, the media was supplemented with $400 \mu \mathrm{g} /$ ml G418 (Invitrogen). Two weeks later, the clones were picked and grown in six-well plates until confluency. Stable SCC9-pEGFP-N1 (SCC9 cells transfected with an empty vector (SCC9-N)) and SCC9-pEGFP-N1-Snail (SCC9 cells transfected with a vector encoding human Snail (SCC9-S)) cell lines were obtained and subcultured.

\section{Quantitative Real-Time PCR}

The total RNA was extracted using the TRIzol reagent (Invitrogen), and the resulting mRNA was reverse transcribed into cDNA using the $5 \times$ PrimeScript RT Master Mix (Ta$\mathrm{KaRa}$ ) at $37^{\circ} \mathrm{C}$ for $15 \mathrm{~min}$ and $85^{\circ} \mathrm{C}$ for $5 \mathrm{~s}$, according to the manufacturer's protocol. Quantitative PCR (qPCR) was performed using $2 \times$ SYBR Premix Ex Taq (TaKaRa) with a 7300 ABI Real-Time PCR System (Applied Biosystems, Foster City, CA, USA) under the following conditions: $95^{\circ} \mathrm{C}$ for $30 \mathrm{~s}, 95^{\circ} \mathrm{C}$ for $5 \mathrm{~s}$, and $60^{\circ} \mathrm{C}$ for $31 \mathrm{~s}$ for 40 cycles. The relative mRNA levels were analysed by the $2(-\Delta \Delta \mathrm{Ct})$ method with GAPDH as an internal control. ${ }^{23}$ GAPDH (5'-GAAGGTGA AGGTCGGAGTC- ${ }^{\prime}, \quad 5^{\prime}$-GAGATGGTGATGGGATTTC- ${ }^{\prime}$ ), E-cadherin $\quad\left(5^{\prime}\right.$-TACACTGCCCAGGAGCCAGA-3' ${ }^{\prime}, \quad 5^{\prime}$-TG GCACCAGTGTCCGGATTA- $\left.3^{\prime}\right)$, Snail (5'-GACCACTATGC CGCGCTCTT- $3^{\prime}$, 5'-TCGCTGTAGTTAGGCTTCCGATT- ${ }^{\prime}$ ), $\beta$-catenin (5'-GCTGAAGGTGCTATCTGTCTGCTC-3', $5^{\prime}$-TG AACAAGACGTTGACTTGGATCTG- $3^{\prime}$ ), vimentin ( $5^{\prime}$-TGAGT ACCGGAGACAGGTGCAG-3'， 5'-TAGCAGCTTCAACGGC AAAGTTC- $3^{\prime}$ ).

\section{Western Blotting}

The cells were grown to $80-90 \%$ confluency in $25 \mathrm{~cm}^{2}$ cell culture flasks (Corning). The whole-cell lysates were collected and their concentrations determined using the Bradford assay. Protein $(20 \mu \mathrm{g})^{24}$ was separated by SDS-PAGE and transferred to PVDF membranes (Millipore, Billerica, MA, USA). The blots were then probed with antibodies, such as anti- $\beta$-actin (1:1000, Santa Cruz Biotechnology, Santa Cruz, CA, USA), anti-E-cadherin (1:400, Santa Cruz Biotechnology), anti- $\beta$-catenin (1:500, Santa Cruz Biotechnology), antivimentin (1:500, Santa Cruz Biotechnology), and anti-Snail (1:500, Abcam, Cambridge, MA, USA). The membranes were developed by Immun-Star WesternC Kit (Bio-Rad, Hercules, CA, USA) products and bands were detected by exposure to film (Kodak, Japan). The relative protein levels were quantified by dividing the other bands by the $\beta$-actin bands.

\section{Immunofluorescence Microscopy}

The SCC9-N and SCC9-S cells were grown on glass coverslips, fixed with $4 \%$ paraformaldehyde (PFA) ${ }^{25}$ permeabilised in $1 \%$ Triton X-100 for 15 min, blocked with $1 \%$ bovine 
serum albumin for $30 \mathrm{~min}$, incubated with antibodies specific for E-cadherin (1:100, Santa Cruz Biotechnology) and vimentin (1:100, Santa Cruz Biotechnology) or Snail (1:100, Abcam), followed by the appropriate secondary antibodies (1:50), and then further incubated with DAPI (1:1000, Invitrogen) for $2 \mathrm{~min}$. The preparations were visualised using a Zeiss LSM-710 laser-scanning confocal microscope.

\section{Wound-Healing and Invasion Assays}

The cells were cultured in six-well plates to $90 \%$ confluency and scraped with a $10-\mu \mathrm{l}$ pipette tip in the central area. After wounding, the detached and damaged cells were carefully removed with phosphate-buffered solution (PBS), and the culture was changed to a serum-free medium. The wound closure was observed microscopically and photographed at the indicated time points. An analysis assessing the invasion of cells was performed using Transwell filters with $6.5-\mathrm{mm}$ diameters and $8-\mu \mathrm{M}$ pore sizes (Costar, Lowell, MA, USA). The filters were precoated for $30 \mathrm{~min}$ at $37^{\circ} \mathrm{C}$ with $25 \mu \mathrm{l}$ extracellular matrix (Sigma-Aldrich) gel mixed with dimethyl sulfoxide (1:1). The trypsinised cells $\left(4 \times 10^{5}\right)$ were washed with PBS, resuspended in the serum-free medium, and placed in the upper chamber, and a medium containing 10\% FBS was used as a chemoattractant in the lower chamber. After incubating for $24 \mathrm{~h}$ at $37^{\circ} \mathrm{C}$ in $5 \% \mathrm{CO}_{2}$, the cells were fixed in $4 \%$ PFA and stained with crystal violet (Sigma-Aldrich) for $30 \mathrm{~min}$. The nonmigratory cells on the upper chamber were removed with cotton swabs, and the migratory cells present on the lower surface were counted in 10 random fields and photographed by field at $\times 100$ magnification under an inverted microscope (Olympus).

\section{Adhesion Assay}

The cells were counted after trypsinisation, and a total of $4 \times 10^{5}$ cells were plated per well for 1 or $2 \mathrm{~h}$, respectively. The unattached cells were carefully removed by washing twice with PBS, and the attached cells were trypsinised again and counted under an inverted microscope (Olympus).

\section{Flow Cytometry}

For the cell cycle analysis, the cultured SCC9-N and SCC9-S cells were detached with trypsin at $37^{\circ} \mathrm{C}$, washed twice with cold PBS, resuspended in $1 \mathrm{ml}$ of $75 \%$ alcohol to ensure that the cells were single-fixed, and then stored at $-20^{\circ} \mathrm{C}$ for $24 \mathrm{~h}$. The prepared cells were washed with PBS again, stained with $100 \mathrm{mg} / \mathrm{ml}$ of propidium iodide (BD Pharmingen, San Jose, CA, USA) at $4{ }^{\circ} \mathrm{C}$ for $30 \mathrm{~min}$, and analysed by flow cytometry using the BD FACS Calibur (BD Biosciences) and CellQuest Pro software (BD Biosciences). Except for the cell-cycle markers, the expression of CSC-like markers was also evaluated by flow cytometry. The cells were collected, resuspended in PBS containing 3\% FBS, and labelled with human-fluorochrome-conjugated anti-CD24-PE (10 $\mu \mathrm{l}$ per test, Beckman Coulter, Los Angeles, CA, USA), anti-CD44PE-Cy5 (0.625 $\mu$ l per test, eBioscience, San Diego, CA, USA), or anti-CD133-PE (10 $\mu$ l per test, Miltenyi Biotech, Auburn, CA, USA) for $30 \mathrm{~min}$ in the dark. The corresponding mouse immunoglobulins conjugated to PE or PE-CY5 (BD Pharmingen) were used as isotype controls in each experiment.

\section{Colony-Forming Assays}

Single-cell suspensions (2000 cells per dish) were seeded on $100-\mathrm{mm}$ dishes (Corning) and cultured for 14 days. The media was changed every 4 days. The colonies were washed twice with PBS, fixed in 4\% PFA, and stained with crystal violet overnight. The colonies were observed and counted under an upright microscope (Olympus). Aggregates of $>50$ cells were defined as a colony according to the system described by Ma et al. ${ }^{26}$

\section{Statistical Analysis}

The data were represented as mean \pm s.d. from at least three independent experiments, ${ }^{27}$ and analysed by independentsamples $t$-test using the SPSS 13.0. software (SPSS). $P$-value less than 0.05 was considered significant.

\section{RESULTS}

\section{Overexpression of Snail Correlates with EMT}

Snail has been shown to induce EMT, during which epithelial cells lose their polarity and convert to a mesenchymal phenotype. ${ }^{16}$ In this study, we transfected SCC9 cells with either an empty vector (SCC9-N) or a vector encoding human Snail (SCC9-S). Six weeks later, the stable SCC9-N and SCC9-S cell lines were obtained. Upon observation by microscopy, the SCC9-S cells showed typical fibroblast-like and spindleshaped appearances, whereas the SCC9-N cells showed robust cellular junctions with typical 'cobblestone-shaped' and epithelial-like appearances (Figure 1a). To determine if the pattern of gene expression correlated with EMT, real-time PCR was performed. The results showed that in the SCC9-S cells, 1.8-fold Snail overexpression decreased the E-cadherin message levels by 1.7 -fold $(P<0.01)$ and downregulated $\beta$-catenin expression by 2 -fold $(P<0.01)$. In addition, the mesenchymal marker vimentin showed a 1.9-fold $(P<0.05)$ increase in mRNA levels compared with the SCC9-N cells (Figure 1b). Then, western blot analysis was examined. The increased expression of Snail (2.91-fold) in the SCC9-S cells resulted in a decrease of the epithelial markers E-cadherin (1.96-fold) and $\beta$-catenin (4.35-fold), but an increase of vimentin (3.11-fold, Figures 1c and d), which was consistent with the real-time PCR results. In addition, the cellularstaining pattern of E-cadherin and vimentin were examined between the two cell lines. As shown by the immunofluorescence analysis, the SCC9-S cells presented a fibroblastlike appearance, with decreased E-cadherin expression but increased vimentin staining. By contrast, the SCC9-N cells possessed an epithelial-like morphology and stained positively for E-cadherin, but weakly for vimentin (Figure 2). These results demonstrated that increased Snail expression was associated with the EMT in SCC9 cells. 


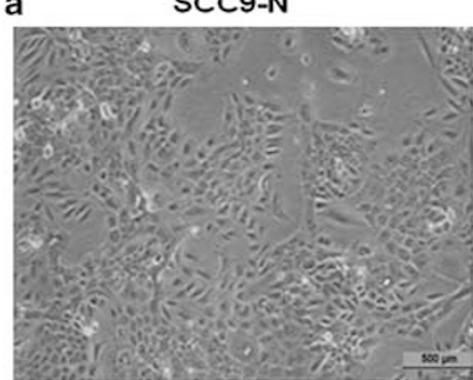

scc9-s

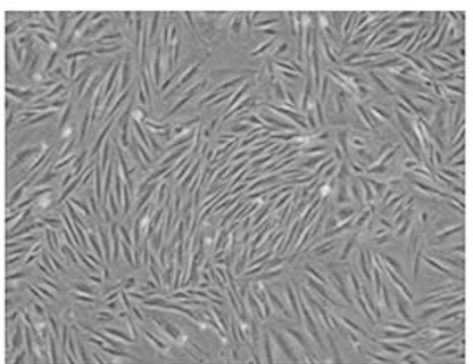

c

\section{E-cadherin}

$\beta$ - catenin

Snai

vimentin

$\beta$ - actin
SCC9-S
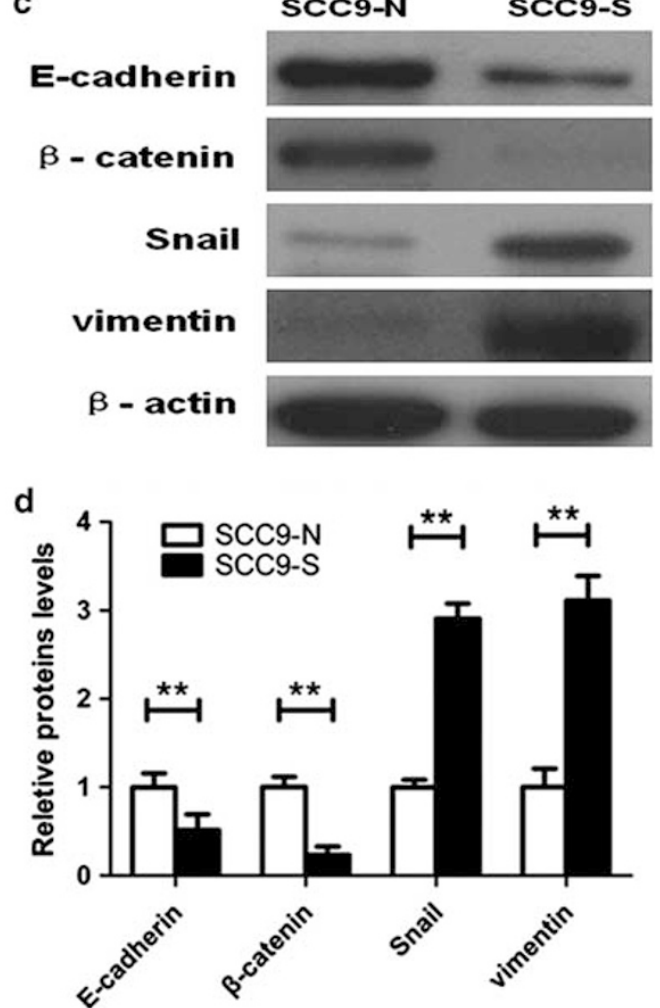

b

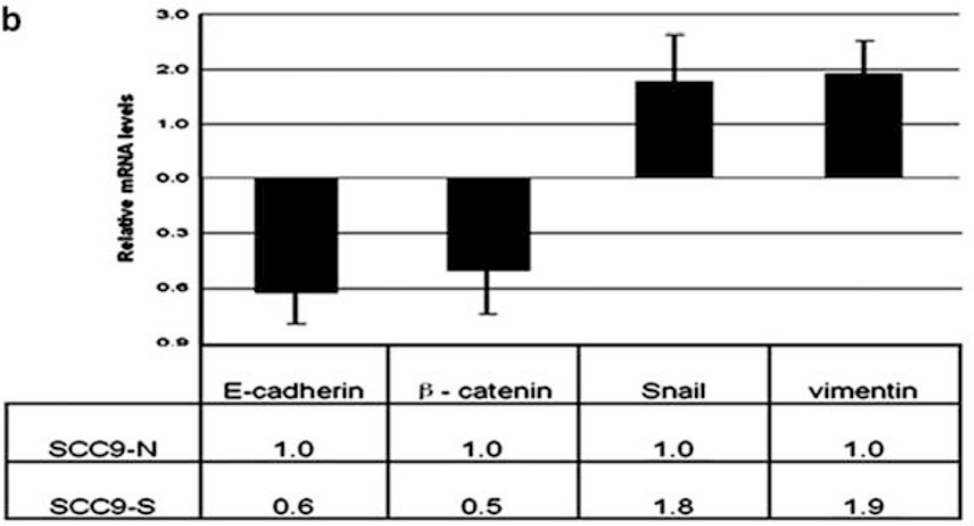

Figure 1 The overexpression of Snail significantly induces the epithelial to mesenchymal transition (EMT). (a) The SCC9 cells transfected with an empty vector (SCC9-N) presented typical cobblestone and epithelial-like appearances. The SCC9 cells transfected with Snail (SCC9-S) had a more elongated, fibroblast-like morphology compared with the controls. The photographs were taken at $\times 40$ magnification, and the scale bar $=500 \mu \mathrm{m}$. (b) A real-time RTPCR was performed to determine the relative mRNA expression levels of E-cadherin, $\beta$-catenin, Snail, and vimentin, the housekeeping gene GAPDH was used as the control. The data were reported as mean \pm s.d. (c) The western blot analysis of the EMT-related proteins. E-cadherin, $\beta$-catenin, vimentin, and Snail were examined, $\beta$-actin was used as a loading control. (d) The relative proteins levels with E-cadherin, $\beta$-catenin, Snail, and vimentin. The data were reported as mean \pm s.d. ${ }^{*} P<0.01$
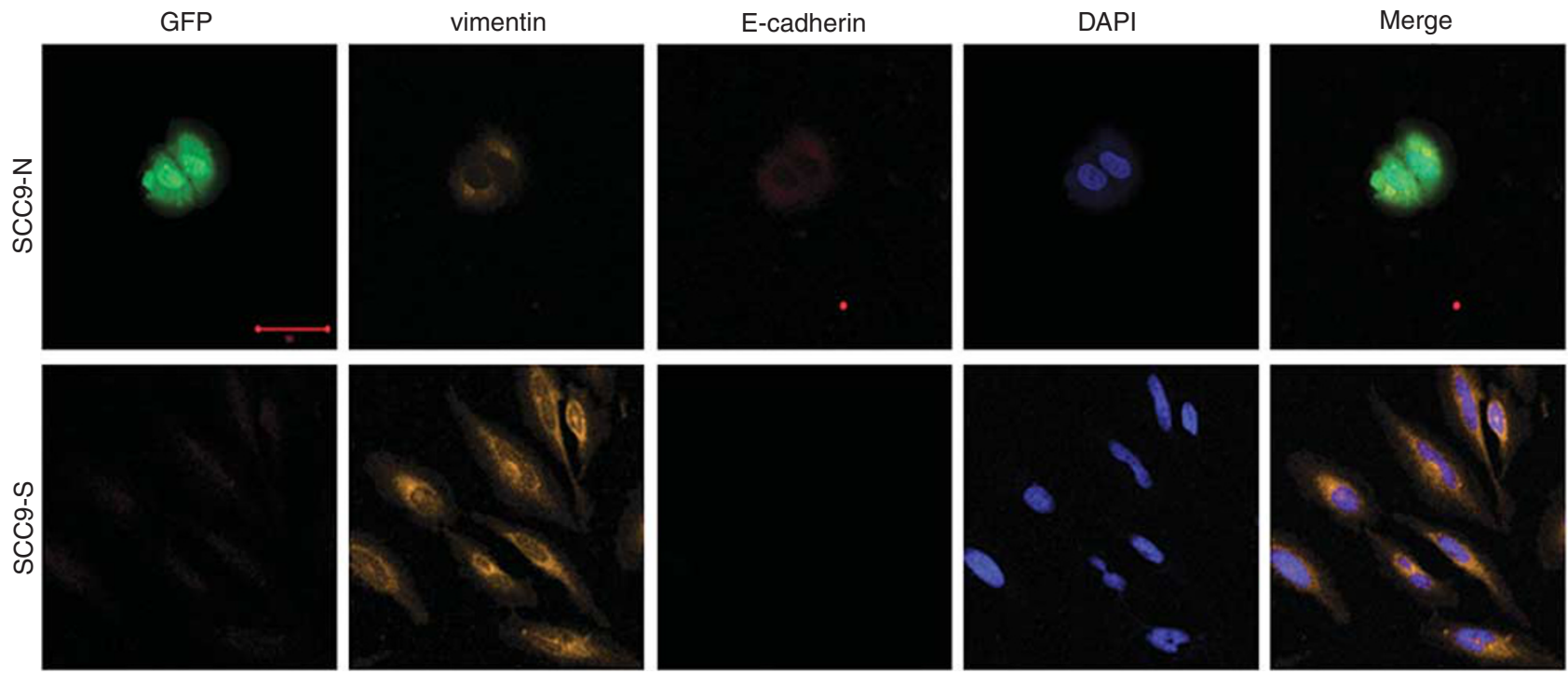

Figure 2 The double immunofluorescence staining of E-cadherin (red) and vimentin (yellow) in the SCC9 cells transfected with an empty vector (SCC9-N) and vector encoding human Snail (SCC9-S) cells. The end of the full-length CDNA for Snail was encoded with the terminator TGA, which blocked the expression of GFP (green) in the SCC9-S cells. The nuclei were stained with DAPI. The increasing expression of Snail in the SCC9-S cells induced a long, spindle-shaped morphology, and exhibited high vimentin expression, whereas the cells were negative for E-cadherin ( $\times 200$ magnification). The scale bar $=50 \mu \mathrm{m}$. 


\section{The Cell Lines that Acquire an EMT Phenotype Show Highly Invasive and Metastatic Behaviour In Vitro}

To test whether the mesenchymal cells were highly invasive and metastatic, Transwell assays were performed to measure single-cell invasion. After $24 \mathrm{~h}$, the invasive cells were quantified and photographed at $\times 100$ magnification in 10 randomly chosen fields. The SCC9-S cells were more invasive than the SCC9-N cells $(P<0.01$, Figure $3 \mathrm{a})$ and showed a 4.9fold increase in gel invasion (Figure $3 \mathrm{~b}$ ), which indicated that enhanced Snail expression contributes to this process. Additionally, the SCC9-S cells had lower adhesive ability than the SCC9-N cells (Figure 3c). The percentage of attached cells was $13.91 \%$ for the SCC9-S and $17.34 \%$ for the SCC9-N. One hour later, the percentage of attached cells increased in both cell lines to 16.09 and $29.06 \%$, respectively. This phenomenon was consistent with our observation that the SCC9-S cells were more easily trypsinised than the SCC9-N cells during subculturing. Furthermore, a wound-healing assay was used to assess the migration of the cells. The results showed that in contrast to the SCC9-N cells, the SCC9-S cells migrated less frequently and could not grow to confluency after $48 \mathrm{~h}$ (Figure 4 ).

\section{Mesenchymal Cells Exhibit CSC-Like Properties}

In this study, a cell-cycle analysis and colony-forming assay were performed to test the proliferative ability. As anticipated, the SCC9-S cells presented a significantly higher percentage of cells in the G0/G1 phases (64.75\%) than did the SCC9-N cells $(51.94 \%)$, but a lower percentage of cells in S phase $(14.02 \%)$ than did the SCC9-N (32.91\%, Figure 5a).
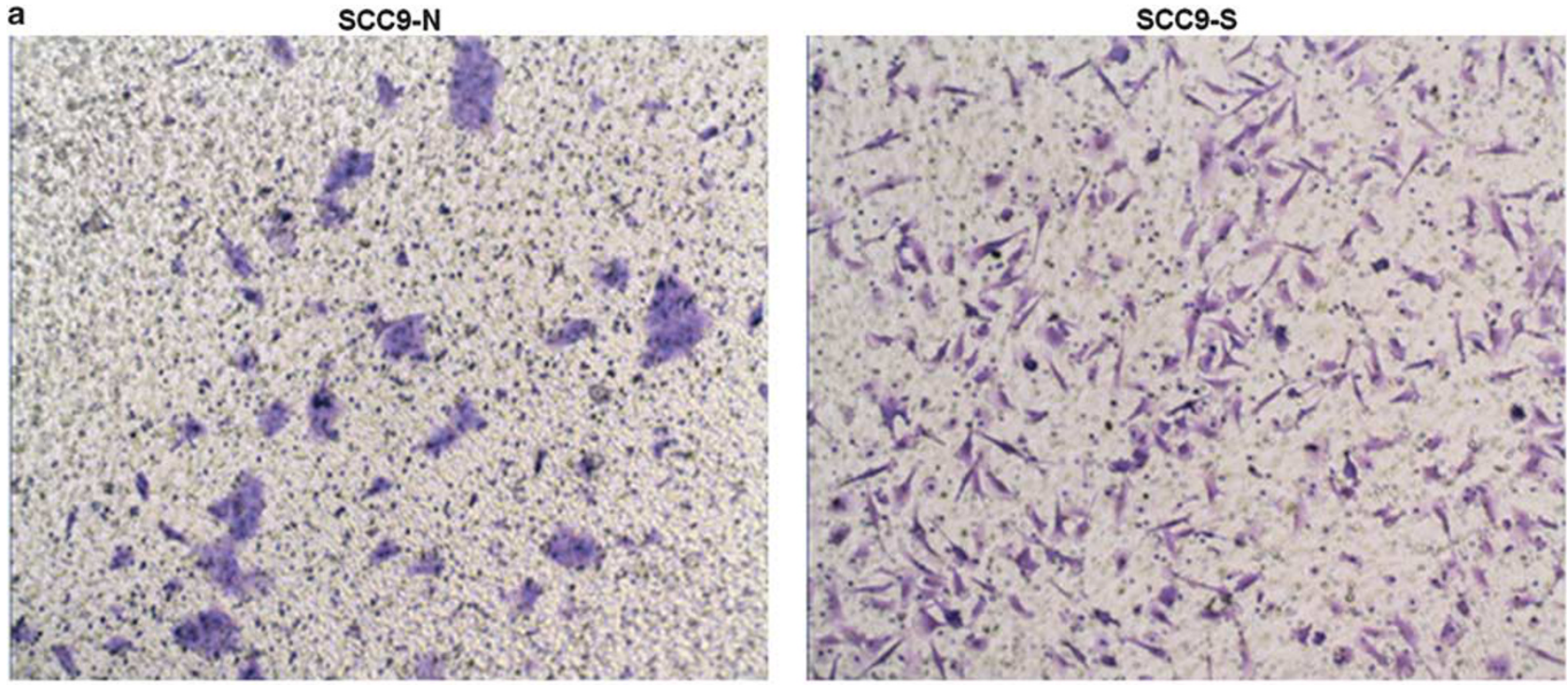

b

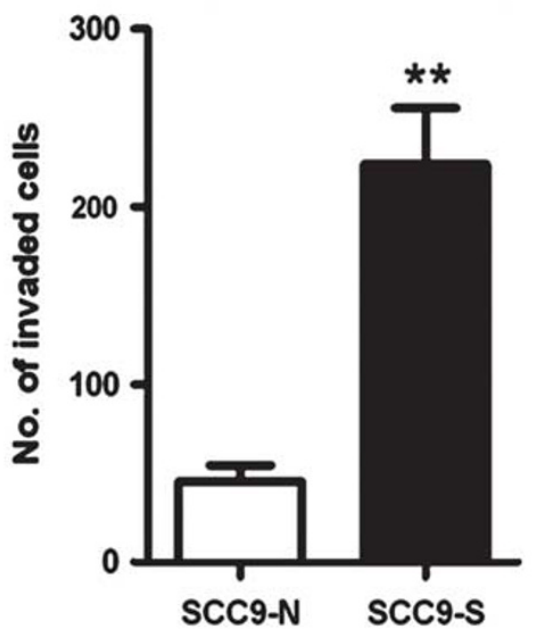

C

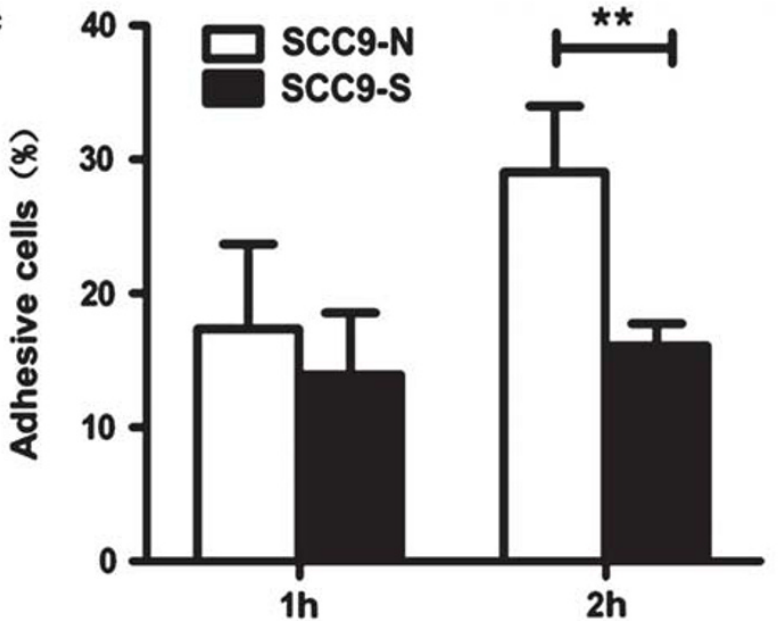

Figure 3 The SCC9-S cells that undergo the epithelial to mesenchymal transition (EMT) are associated with highly invasive and metastatic behaviour. (a) The cells invading the filters coated with the extracellular matrix (ECM) gel. The photographs were taken at $\times 100$ magnification. (b) The quantification of the cell invasion in 10 randomly chosen fields. The data were reported as mean \pm s.d. ${ }^{* *} P<0.01$ compared with that of SCC9 cells transfected with an empty vector (SCC9-N) cells. (c) The quantification of cell adhesion at two time points. The data were reported as mean \pm s.d. ${ }^{\star \star P}<0.01$. 

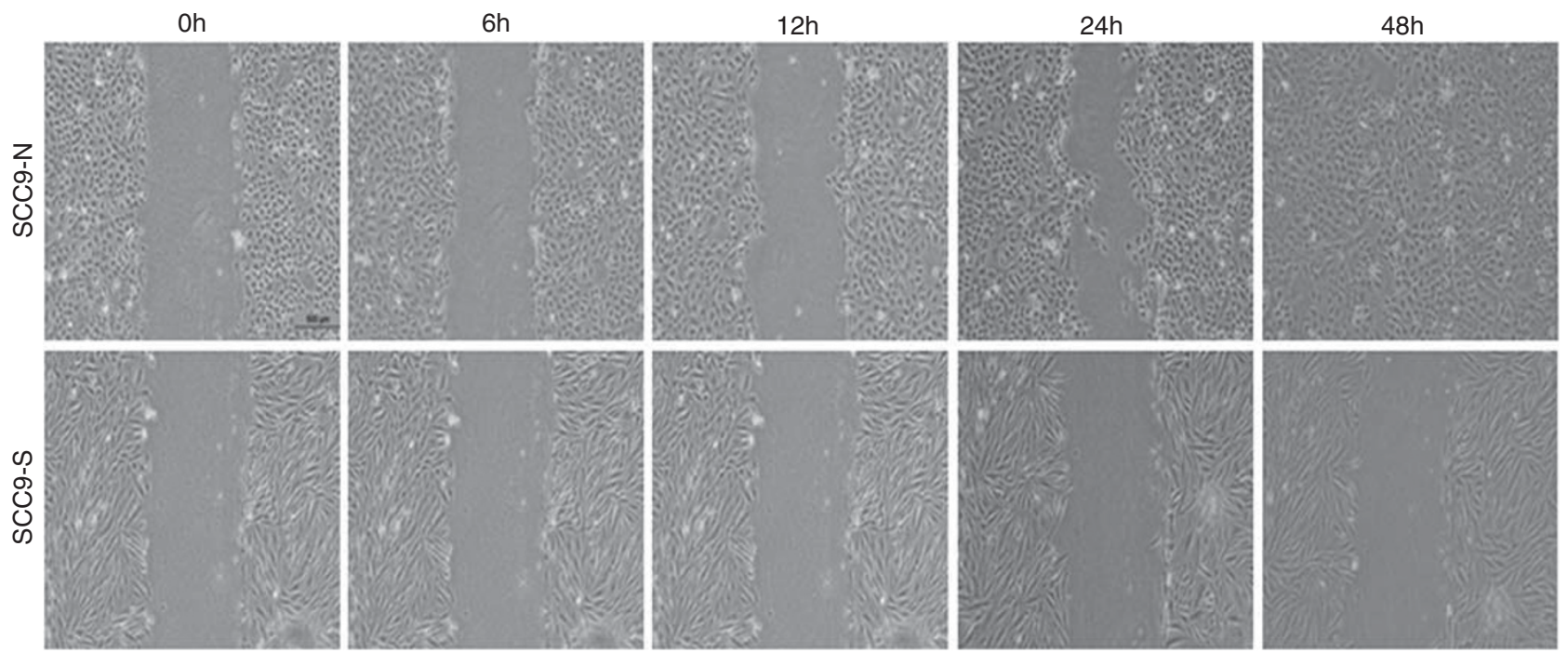

Figure 4 The capacity of the cells to fill the scratched area was examined under microscopy at the indicated time points. Confluent cell monolayers were scraped by a pipette tip in the central area. The SCC9 cells transfected with a vector encoding human Snail (SCC9-S) cells did not close the wound after $48 \mathrm{~h}$. The photographs were taken at $\times 40$ magnification, and the scale bar $=500 \mu \mathrm{m}$.

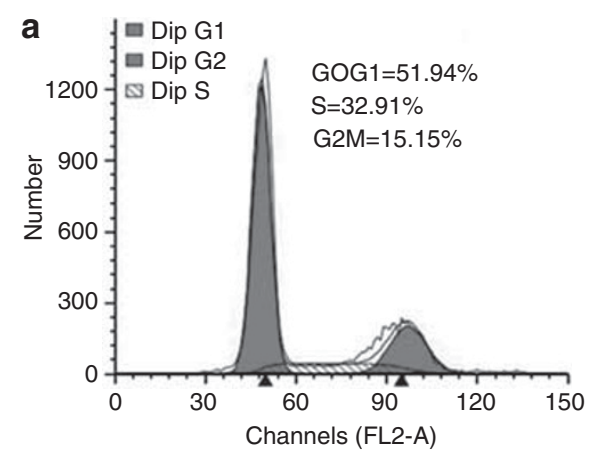

b

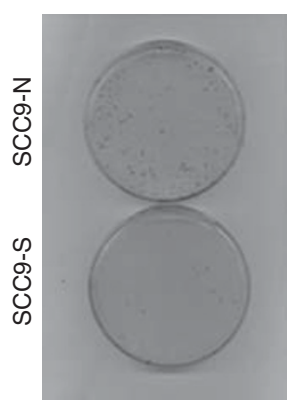

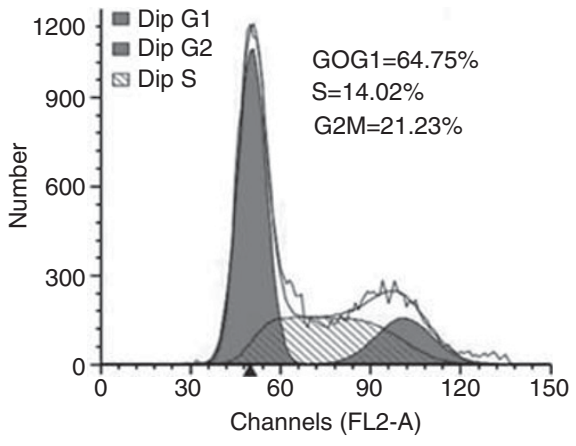

d

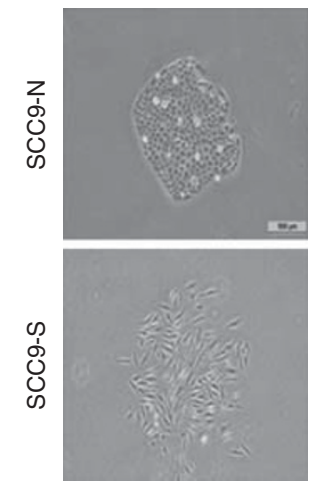

Figure 5 The SCC9 cells transfected with a vector encoding human Snail (SCC9-S) cells present low proliferation, but are self-renewable. (a) In the SCC9 cells transfected with an empty vector (SCC9-N) cells, 51.94\% of the cells were arrested in the G0/G1-phase, in the SCC9-S cells, 64.75\% of the cells were arrested in the G0/G1-phase, indicating that cell mitosis was blocked at the G0/G1 checkpoint and cell proliferation was inhibited. (b) Images of colonies stained with crystal violet. (c) The quantification of the colonies formed by the two cells, indicating that the SCC9-S cells had low proliferation ability. The data were reported as mean \pm s.d. ${ }^{* * P}<0.01$ compared with that of SCC9-N cells. (d) The SCC9-S cells can form colonies, indicating that they are self-renewable. The photographs were taken at $\times 40$ magnification, and the scale bar $=500 \mu \mathrm{m}$.

The colony-forming assay presented that the SCC9-S cells proliferated less frequently (Figure $5 \mathrm{~b}$ ), showed a 5.0 -fold decrease in colony formation compared with the SCC9-N cells $(P<0.01$, Figure $5 \mathrm{c})$. In addition to using proliferation ability, self-renew ability was also measured by the colonyforming assay. Although the SCC9-S cells displayed low proliferative ability, they showed self-renewal that can maintain the population of tumorigenic cells (Figure 5d). By 
a
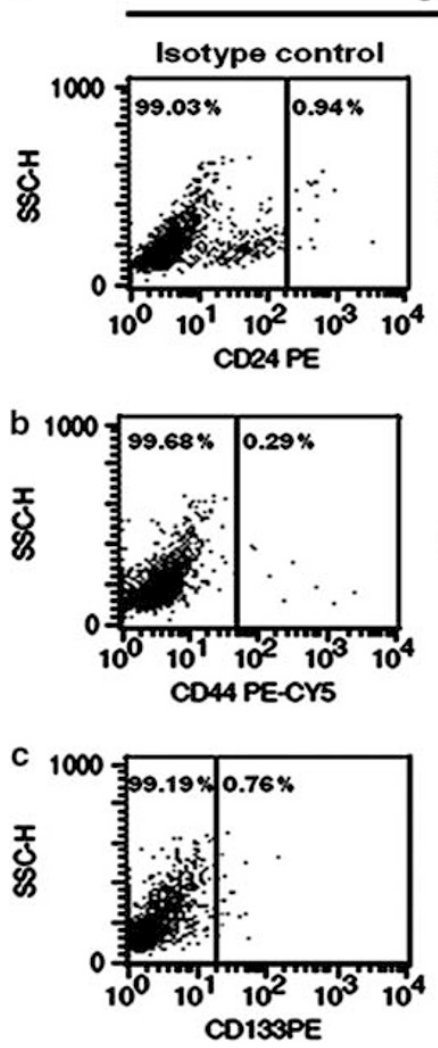

SCC9-N
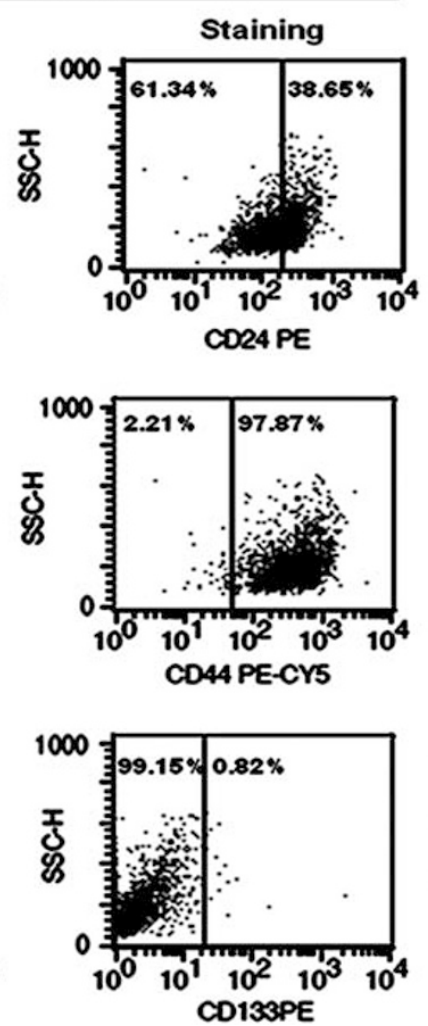

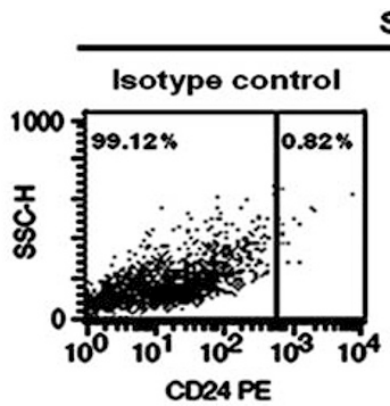

SCC9-S
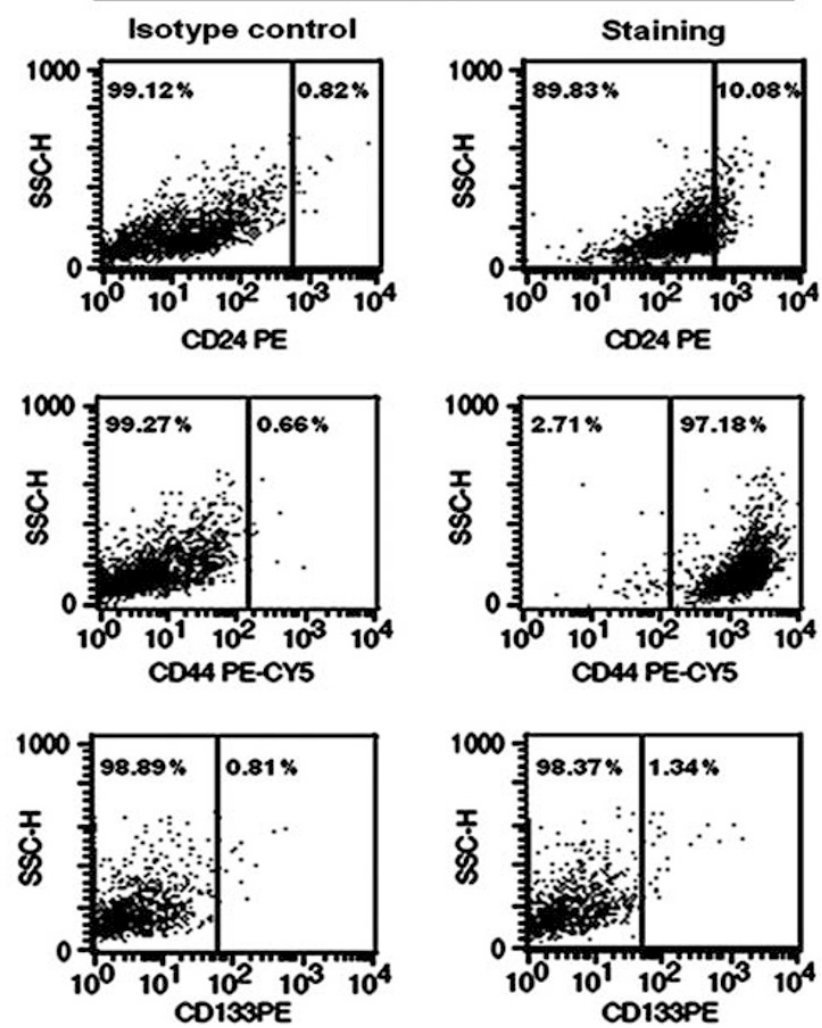

Figure 6 The overexpression of Snail generates a cancer stem cell (CSC)-like phenotype in SCC9 cells transfected with a vector encoding human Snail (SCC9-S) cells. The flow cytometry analysis of the CD24, CD44, and CD133 expression in the SCC9-S and SCC9 cells transfected with an empty vector (SCC9N) cells. (a) The expression of CD24 was downregulated in the SCC9-S cells. (b) A high level of CD44 expression was assessed in the two cell lines. (c) CD133 expression was not detected in the two cell lines. Mouse immunoglobulins conjugated to PE or PE-CY5 were used as isotype controls in each experiment.

extension, we speculated that Snail-induced cells that undergo EMT may have stem cell-like properties. Then, CSCs surface markers expression for these cells was examined using flow cytometry. As shown in Figure 6, CD24 expression was lower in the SCC9-S cells (9.26\%) than in the SCC9-N cells (37.71\%, Figure 6a). However, there was no significant difference in CD44 expression between the two cell lines, the percentage of SCC9-S cells expressing CD44 was $96.52 \%$, and in SCC9-N cells, it was $97.58 \%$ (Figure 6b), and in this study, SCC9-N and SCC9-S cells showed no significant CD133 expression (Figure 6c). Thus, the SCC9-S cells presented as CD133-negative and CD44 $4^{\text {high }} / \mathrm{CD} 24^{\text {low }}$, and the SCC9-N cells presented as CD133-negative and $\mathrm{CD} 44^{\text {high }} / \mathrm{CD} 24^{+}$.

\section{DISCUSSION}

OSCC is a highly invasive and an aggressive cancer. ${ }^{1}$ Recently, the EMT has been found to contribute to the progression and confer cancer cells with stem cell-like properties, in which the cells have a propensity for invasion, metastatic dissemination, resistance to certain therapy, persistence in tumours, and recurrence. ${ }^{19,28}$ Understanding the molecular and cellular changes may generate novel targets that can overcome the aforementioned issues and reduce mortality. In this study, we made three novel observations that may help develop new treatments for OSCC: overexpression of Snail induced the SCC9 cells to undergo EMT; furthermore, these cells exhibited mesenchymal properties and were highly invasive, but not highly proliferative; and the induction of EMT conferred CSC-like properties to these mesenchymal cells.

In this study, the SCC9 cells that overexpressed Snail adopted mesenchymal cell properties. SCC9-S cells presented fibroblastoid phenotypes, had a more extended and spindlelike shape than did the SCC9-N cells. These significant morphological changes combined with the functional analysis demonstrated that the epithelial tumour cells underwent the EMT, in which the epithelial markers (such as E-cadherin and $\beta$-catenin) were downregulated and the mesenchymal markers (such as vimentin) were upregulated. Importantly, the SCC9-S cells acquired high invasive properties in our Transwell analysis. Thus, overexpression of Snail not only triggered the EMT in SCC9 cells, but also promoted its progression. Our findings in SCC9 cells are consistent with the prior studies have shown that Snail has a crucial role in EMT and is associated with an invasive ability in various human cancers. ${ }^{1,12,13,29-31}$ 
EMT was also accompanied by the losing of cell adhesion and an increasing in cell motility. ${ }^{32}$ As shown in adhesion assays, overexpression of Snail inhibited cell-cell contacts. The SCC9-S cells exhibited a scattered phenotype and loss of adhesion, which contributes to cells becoming motile and correlates with their metastasis. However, the wound-healing assays revealed the novel results. The SCC9-S cells were highly invasive and metastatic, but they did not migrate into the scratched area after $48 \mathrm{~h}$ in culture due to their limited proliferation. This finding was consistent with the cell-cycle analysis, showing that the cell mitosis was blocked at the G0/ G1 checkpoint and reduced in the $\mathrm{S}$ phase, which decreased the rate of cell proliferation in SCC9-S cells. In other words, Snail could be considered to be a master gene in the EMT, and led to invasive properties ${ }^{13,33}$ but low proliferation rates in the SCC9-S cells. Such novel results are supported by a previous study that shows Snail-expressing epithelial cells that have undergone EMT have a low proliferation potential. ${ }^{18,34,35}$ Our findings also present a likely explanation for the increased invasion, but decreased proliferation in the EMT: the SCC9-S cells are relatively quiescent, and composed of many cells in G0 phase that divide infrequently and may intrinsically resistant to current drug and radiotherapies designed to inhibit rapidly dividing cells. ${ }^{20}$ On the basis of the current study, we speculate that overexpression of Snail in SCC9 cells induces the EMT and promotes CSC-like properties in mesenchymal cells, which correlates with the conclusion of Mani et $a l^{22}$ and is also supported by Santisteban $e t a l,{ }^{36}$ suggesting that the in vitro induction of EMT can generate cancer stem-cell properties from differentiated epithelial tumour cells.

Self-renewal, which is one of the hallmarks of CSCs, has been demonstrated in CSCs through their ability to form colonies. ${ }^{20,21}$ In this study, though the SCC9-S cells grew slowly in the serum-free culture media, they were able to form colonies, which is consistent with stem-cell properties. Moreover, the SCC9-S cells exhibited a CD24 $4^{\text {low }}$ expression, but $\mathrm{CD}_{24}{ }^{+}$expression in the SCC9-N cells, showed that 'stemness' is associated with CD24 ${ }^{-/ \text {low }}$ cells. ${ }^{36}$ Prior studies showed that CD44 and CD133 had been used to isolate CSCs from multiple tumor types. ${ }^{21,37-39}$ However, in the present study, both of the cell lines presented as CD44 $4^{\text {high }}$, and showed only $0.53 \%$ of the SCC9-S cells expressed CD133, in contrast to $0.06 \%$ of the SCC9-N cells. With these findings in mind, a 'stemness' marker that works well in one cancer may not apply to other types of cancers. ${ }^{20}$ It would be worthwhile to explore the values of CD44 and CD133 in combination with other cell surface markers for efficiently isolating CSCs $^{21,40}$ in SCC9 cells.

In conclusion, SCC9 cells that overexpressed Snail undergo EMT, which presents mesenchymal phenotype and leads to invasive and metastatic properties. Furthermore, the mesenchymal cells show low proliferation rates, but still have self-renewal ability, and present decreased CD24 expression. These findings indicate that there is a link between the EMT and CSCs in SCC9 cells. Importantly, Snail has a critical role in this process. Further exploring this mechanism may not only provide new insight into oral cancer research, but also identify a useful molecular target for efficiently treating this disease.

\section{ACKNOWLEDGEMENTS}

We thank Sheng Chen and Wei Zhang for their excellent technical assistance. This study was supported by Grants from the National Natural Science Foundation of China (Grant No. 30872903 and 30801301) and from the Project of Science and Technology Department of the Jiangsu Province (Grant No. BK2008362). We also thank the American Journal Experts for revising the English used in this article.

\section{DISCLOSURE/CONFLICT OF INTEREST}

The authors declare no conflict of interest.

1. Sun L, Diamond ME, Ottaviano AJ, et al. Transforming growth factorbeta 1 promotes matrix metalloproteinase-9-mediated oral cancer invasion through snail expression. Mol Cancer Res 2008;6:10-20.

2. Ramos DM, Dang D, Sadler S. The role of the integrin alpha v beta 6 in regulating the epithelial to mesenchymal transition in oral cancer. Anticancer Res 2009;29:125-130.

3. Silverman Jr S. Demographics and occurrence of oral and pharyngeal cancers. The outcomes, the trends, the challenge. J Am Dent Assoc 2001;132Suppl:7S-11S.

4. Prince $M E$, Sivanandan $R$, Kaczorowski $A$, et al. Identification of a subpopulation of cells with cancer stem cell properties in head and neck squamous cell carcinoma. Proc Natl Acad Sci USA 2007;104: 973-978.

5. Crnic I, Christofori G. Novel technologies and recent advances in metastasis research. Int J Dev Biol 2004;48:573-581.

6. Molloy T, van 't Veer LJ. Recent advances in metastasis research. Curr Opin Genet Dev 2008;18:35-41.

7. Wu Y, Zhou BP. New insights of epithelial-mesenchymal transition in cancer metastasis. Acta Biochim Biophys Sin (Shanghai) 2008;40: 643-650.

8. Thiery JP. Epithelial-mesenchymal transitions in development and pathologies. Curr Opin Cell Biol 2003;15:740-746.

9. Cardiff RD. Epithelial to mesenchymal transition tumors: Fallacious or Snail's Pace? Clin Cancer Res 2005;11(24 Pt 1):8534-8537.

10. Thompson EW, Newgreen DF, Tarin D. Carcinoma invasion and metastasis: a role for epithelial-mesenchymal transition? Cancer Res 2005;65:5991-5995; discussion 5995.

11. Thiery JP, Sleeman JP. Complex networks orchestrate epithelialmesenchymal transitions. Nat Rev Mol Cell Biol 2006;7:131-142.

12. Batlle $E$, Sancho $E$, Franci $C$, et al. The transcription factor snail is a repressor of E-cadherin gene expression in epithelial tumour cells. Nat Cell Biol 2000;2:84-89.

13. Cano A, Perez-Moreno MA, Rodrigo I, et al. The transcription factor snail controls epithelial-mesenchymal transitions by repressing $\mathrm{E}$ cadherin expression. Nat Cell Biol 2000;2:76-83.

14. Wu Y, Zhou BP. Snail: more than EMT. Cell Adh Migr 2010;4:199-203.

15. Takkunen M, Grenman R, Hukkanen M, et al. Snail-dependent and independent epithelial-mesenchymal transition in oral squamous carcinoma cells. J Histochem Cytochem 2006;54:1263-1275.

16. Yang $\mathrm{MH}, \mathrm{Chang} \mathrm{SY}, \mathrm{Chiou} \mathrm{SH}$, et al. Overexpression of NBS1 induces epithelial-mesenchymal transition and co-expression of NBS1 and Snail predicts metastasis of head and neck cancer. Oncogene 2007;26: 1459-1467.

17. Higashikawa K, Yoneda S, Taki M, et al. Gene expression profiling to identify genes associated with high-invasiveness in human squamous cell carcinoma with epithelial-to-mesenchymal transition. Cancer Lett 2008;264:256-264.

18. Peinado $H$, Olmeda D, Cano A. Snail, Zeb and bHLH factors in tumour progression: an alliance against the epithelial phenotype? Nat Rev Cancer 2007;7:415-428. 
19. Polyak K, Weinberg RA. Transitions between epithelial and mesenchymal states: acquisition of malignant and stem cell traits. Nat Rev Cancer 2009;9:265-273.

20. Clarke MF, Dick JE, Dirks PB, et al. Cancer stem cells-perspectives on current status and future directions: AACR Workshop on cancer stem cells. Cancer Res 2006;66:9339-9344.

21. Hurt EM, Farrar WL. Cancer Stem Cells. Cambridge University Press: New York, 2010.

22. Mani SA, Guo W, Liao MJ, et al. The epithelial-mesenchymal transition generates cells with properties of stem cells. Cell 2008; 133:704-715.

23. Waldmann J, Slater EP, Langer $\mathrm{P}$, et al. Expression of the transcription factor snail and its target gene twist are associated with malignancy in pheochromocytomas. Ann Surg Oncol 2009;16:1997-2005.

24. Vasko V, Espinosa AV, Scouten W, et al. Gene expression and functional evidence of epithelial-to-mesenchymal transition in papillary thyroid carcinoma invasion. Proc Natl Acad Sci USA 2007; 104:2803-2808.

25. Jo M, Eastman BM, Webb DL, et al. Cell signaling by urokinase-type plasminogen activator receptor induces stem cell-like properties in breast cancer cells. Cancer Res 2010;70:8948-8958.

26. Ma D, Ma Z, Zhang $X$, et al. Effect of age and extrinsic microenvironment on the proliferation and osteogenic differentiation of rat dental pulp stem cells in vitro. J Endod 2009;35:1546-1553.

27. Giannoni E, Bianchini F, Masieri L, et al. Reciprocal activation of prostate cancer cells and cancer-associated fibroblasts stimulates epithelial-mesenchymal transition and cancer stemness. Cancer Res 2010;70:6945-6956.

28. Clay MR, Tabor M, Owen $\mathrm{JH}$, et al. Single-marker identification of head and neck squamous cell carcinoma cancer stem cells with aldehyde dehydrogenase. Head Neck 2010;32:1195-1201.
29. Sugimachi K, Tanaka S, Kameyama T, et al. Transcriptional repressor snail and progression of human hepatocellular carcinoma. Clin Cancer Res 2003;9:2657-2664.

30. Rosivatz E, Becker I, Specht K, et al. Differential expression of the epithelial-mesenchymal transition regulators snail, SIP1, and twist in gastric cancer. Am J Pathol 2002;161:1881-1891.

31. Moody SE, Perez D, Pan TC, et al. The transcriptional repressor Snai promotes mammary tumor recurrence. Cancer Cell 2005;8:197-209.

32. Gavert N, Ben-Ze'ev A. Epithelial-mesenchymal transition and the invasive potential of tumors. Trends Mol Med 2008;14:199-209.

33. Thiery JP. Epithelial-mesenchymal transitions in tumour progression. Nat Rev Cancer 2002;2:442-454.

34. Vega $\mathrm{S}$, Morales AV, Ocana $\mathrm{OH}$, et al. Snail blocks the cell cycle and confers resistance to cell death. Genes Dev 2004;18:1131-1143.

35. Barrallo-Gimeno A, Nieto MA. The Snail genes as inducers of cell movement and survival: implications in development and cancer. Development 2005;132:3151-3161.

36. Santisteban M, Reiman JM, Asiedu MK, et al. Immune-induced epithelial to mesenchymal transition in vivo generates breast cancer stem cells. Cancer Res 2009;69:2887-2895.

37. Zhou L, Wei X, Cheng $L$, et al. CD133, one of the markers of cancer stem cells in Hep-2 cell line. Laryngoscope 2007;117:455-460.

38. Wei XD, Zhou L, Cheng $L$, et al. In vivo investigation of CD133 as a putative marker of cancer stem cells in Hep-2 cell line. Head Neck 2009;31:94-101.

39. Chen H, Zhou L, Dou T, et al. BMI1'S maintenance of the proliferative capacity of laryngeal cancer stem cells. Head Neck 2011;33:1115-1125 doi:10.1002/hed.21576.

40. Leung EL, Fiscus RR, Tung JW, et al. Non-small cell lung cancer cells expressing CD44 are enriched for stem cell-like properties. PLoS One 2010;5:e14062. 